\title{
Minimally invasive direct coronary artery bypass improves late survival compared with drug-eluting stents in isolated proximal left anterior descending artery disease: A 10-year follow-up, single-center, propensity score analysis
}

\author{
Umberto Benedetto, MD, PhD, ${ }^{a}$ Shahzad G. Raja, MRCS, FRCS(C-Th), ${ }^{a}$

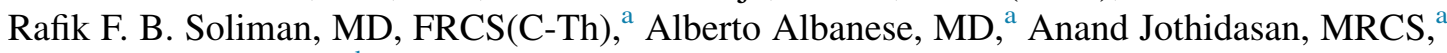 \\ Charles D. Ilsley, MD, ${ }^{b}$ and Mohamed Amrani, MD, PhD, FECTS, ${ }^{a}$ on behalf of the Harefield Cardiac \\ Outcomes Research Group
}

\begin{abstract}
Objectives: Minimally invasive direct coronary artery bypass (MIDCAB) has been proposed to reduce surgical morbidity and improve long-term outcomes compared with stenting in the treatment of isolated proximal left anterior descending artery. However, the survival benefit from MIDCAB still needs to be demonstrated, in particular, because percutaneous coronary intervention with drug-eluting stents (DES-PCI) continues to be considered the initial treatment strategy. We conducted a 10-year follow-up, single-center, propensity score-matched MIDCAB versus DES-PCI comparison.
\end{abstract}

\begin{abstract}
Methods: A total of 1033 patients (303 MIDCAB and 730 DES-PCI) with isolated proximal left anterior descending disease were included. Propensity score matching was used to compare 303 pairs of MIDCAB and DES-PCI patients.
\end{abstract}

Results: MIDCAB and DES-PCI presented with comparable 30-day mortality (2 of 303 [0.6\%] vs 1 of $303[0.3 \%] ; P=1.0$ ). At 10 years, DES-PCI was associated with a 2.19 -fold increased risk of late death (95\% confidence interval, 1.15-4.17), a 2.0 -fold increased risk of repeat revascularization $(95 \%$ confidence interval, 1.20-3.47), and a 2.14-fold increased risk of the composite of death and repeat revascularization (95\% confidence interval, 1.41-3.24).

Conclusions: These findings strongly support a survival benefit from MIDCAB at long-term follow-up compared with DES-PCI in the treatment of isolated left anterior descending disease. (J Thorac Cardiovasc Surg 2014;148:1316-22)

Despite the introduction of drug-eluting stents (DESs), which have reduced the incidence of subsequent restenosis, coronary artery bypass grafting $(\mathrm{CABG})$ remains associated with a decreased risk of repeat revascularization and potentially improved survival at long-term follow-up in patients with multivessel coronary disease. ${ }^{1-4}$

However, percutaneous coronary intervention with drug-eluting stents (DES-PCI) has continued to be widely accepted as the first-line treatment of isolated proximal left anterior descending (LAD) stenosis, and CABG is usually offered for lesions unsuitable for PCI only. ${ }^{5,6}$

The development of minimally invasive direct coronary artery bypass (MIDCAB) using the left internal mammary

From the Departments of Cardiac Surgery ${ }^{\mathrm{a}}$ and Cardiology, ${ }^{\mathrm{b}}$ Harefield Hospital, London, United Kingdom.

Disclosures: Authors have nothing to disclose with regard to commercial support. Received for publication Sept 11,2013; revisions received Nov 20,2013; accepted for publication Dec 10, 2013; available ahead of print Feb 9, 2014.

Address for reprints: Umberto Benedetto, MD, PhD, Department of Cardiac Surgery, Harefield Hospital, London, United Kingdom (E-mail: umberto.benedetto@ hotmail.com).

$0022-5223 / \$ 36.00$

Copyright (c) 2014 by The American Association for Thoracic Surgery

http://dx.doi.org/10.1016/j.jtcvs.2013.12.062 artery (LIMA) to the LAD has emerged as an attractive and less invasive approach than the conventional sternotomy procedure in patients with isolated LAD disease.

Theoretically, arterial revascularization of the LAD has been expected to result in better late outcomes than DES-PCI. This advantage has largely resulted from the subsequent protection of the entire proximal and midLAD artery from future disease progression compared with focal treatment with PCI. ${ }^{8}$

However, the lack of evidence supporting a definitive survival benefit with MIDCAB compared with DES-PCI in the long term has led to even more widespread adoption of percutaneous techniques with concomitant limited MIDCAB popularity and uptake. We aimed to achieve insights into the role of MIDCAB compared with DESPCI in the treatment of isolated proximal LAD disease by conducting a 10-year follow-up, single-center, propensity score-matched cohort comparison.

\section{METHODS}

\section{Study Population}

The present study was conducted in accordance with the principles of the Declaration of Helsinki. The local ethical committee approved the 


\section{Abbreviations and Acronyms \\ $\mathrm{CABG}=$ coronary artery bypass grafting \\ DES = drug-eluting stent \\ LAD $=$ left anterior descending \\ LIMA $=$ left internal mammary artery \\ MIDCAB $=$ minimally invasive direct coronary artery bypass \\ PCI = percutaneous coronary intervention \\ PS $\quad=$ propensity score}

present study, and the requirement for individual patient consent was waived.

We retrospectively analyzed prospectively collected data from the institutional surgical and interventional database (Patient Analysis and Tracking System [PATS]; Dendrite Clinical Systems, Ltd, Oxford, UK). The PATS database captures detailed information on a wide range of preoperative, intraoperative, and hospital postoperative variables (including complications and mortality) for all patients undergoing CABG or PCI at our institution. The data were collected and reported in accordance with the Society for Cardiothoracic Surgery in Great Britain and Ireland database criteria. The database is maintained by a team of full-time clinical information analysts, who are responsible for continuous prospective data collection as a part of a continuous audit process. The data collection is validated regularly. Information about death from any cause is regularly obtained from the General Register Office approximately 1 week after the event. Data on repeat revascularization were obtained from a network of institutional databases.

All patients with a nonemergency status with isolated proximal LAD disease undergoing first-time MIDCAB or DES-PCI from April 2001 to May 2013 were included in the present analysis. The exclusion criteria were admission for acute coronary syndrome and undergoing primary $\mathrm{PCI}$, emergency or salvage $\mathrm{CABG}$, and previous CABG.

In the DES-PCI group, paclitaxel-eluting stents and sirolimus-eluting stents were used. All DES-PCI patients received aspirin (325 mg daily) before and after the procedure and a 300-mg loading dose of clopidogrel the day before the procedure and $75 \mathrm{mg}$ daily for $\geq 3$ months thereafter.

All MIDCAB patients underwent surgery without extracorporeal circulation, and all surgical procedures included implantation of the LIMA to the LAD. In brief, a limited left anterolateral thoracotomy was performed through the fourth intercostal space. The LIMA was harvested either under direct vision or using thoracoscopic assistance. After administration of heparin (200 U/kg), the activated clotting time was kept at $\geq 400$ seconds by repeated application of heparin, if needed. The LIMA was divided distally. Local immobilization of the anastomotic site was achieved with mechanical stabilizers. Anastomosis was performed using 1 running 8-0 polypropylene suture on the beating heart. Protamine was applied to partially neutralize the dose of heparin. The wounds were closed in a standard fashion. In the present cohort, MIDCAB was performed only when the LAD lesions were judged unsuitable for PCI. In most cases, the MIDCAB patients received aspirin $75 \mathrm{mg}$ daily. Dual antiplatelet therapy was not recommended after MIDCAB. Clopidogrel was prescribed for patients with aspirin intolerance. Most MIDCAB procedures (225 of $303[74.2 \%]$ ) were performed by a single surgeon (M.A.) fully experienced in off-pump CABG.

\section{Study Endpoints}

The primary study endpoint was all-cause 10-year mortality; this represented the most robust and unbiased index event because no adjudication was required, thus avoiding inaccurate or biased documentation and clinical assessments. The secondary endpoints were target vessel revascularization, the need for repeat revascularization (PCI or CABG), and the composite of death or repeat revascularization.

Early outcomes were also investigated, including 30-day mortality, hospital length of stay, the incidence of perioperative stroke, and the need for renal replacement therapy. The incidence of local complications was also investigated, including wound infections and arterial complications for MIDCAB and DES-PCI, respectively.

\section{Statistical Analysis}

For baseline characteristics, the variables are summarized as the mean \pm standard deviation for continuous variables and numbers and percentages for categorical variables.

The risk factors investigated were age $\geq 70$ years, gender, hypertension, hypercholesterolemia, New York Heart Association functional class, diabetes mellitus, obesity (defined as a body mass index $\geq 30 \mathrm{~kg} / \mathrm{m}^{2}$ ), serum creatinine $\geq 200 \mathrm{mmol} / \mathrm{L}$, previous myocardial infarction, previous PCI, functional New York Heart Association class III or IV, reduced left ventricular ejection fraction $(<50 \%)$ poor left ventricular function $(<30 \%)$, current smoking, history of cerebrovascular accident, peripheral vascular disease, an elective indication, and the prevalence of chronic total occlusion.

Because of the significant imbalances in baseline covariates between the 2 groups, we used propensity score (PS) matching. A PS representing the probability of undergoing MIDCAB versus DES-PCI was calculated for each patient using nonparsimonious logistic regression model that included all baseline risk factors. The pairs of patients treated by MIDCAB and DES-PCI were derived using greedy 1:1 matching with a caliper width of 0.2 standard deviations of the logit of the PS. A covariate balance was measured using the standardized differences, by which an absolute standardized difference of $>10 \%$ has been suggested to represent a meaningful covariate imbalance.

Generalized linear mixed models, as appropriate, were used to estimate the effect of MIDCAB compared with DES-PCI on the early outcomes.

Kaplan-Meier estimates were used to plot the rates of long-term adverse events (all-cause death, repeat revascularization, and the composite of death or repeat revascularization), and differences between the risk curves were assessed using the Klein-Moeschberger test for matched pairs. ${ }^{9}$ For each adverse outcome, the hazard ratio of MIDCAB versus DES-PCI was estimated using Cox proportional hazard models with robust standard errors to account for clustering in the matched pairs. The consistency of treatment effects across patient characteristics was investigated using a prespecified subgroup analysis (age $\geq 70$ vs $<70$ years; DES-PCI with sirolimus-eluting stents vs paclitaxel-eluting stents; and diabetes mellitus status) for all outcomes of interest. In addition, the covariate interactions, including age, diabetes, and baseline serum creatinine $\geq 200 \mathrm{mmol} / \mathrm{L}$, on the effect estimate for the primary outcomes were tested to identify the subgroup of patients most likely to benefit from MIDCAB. All statistical analyses were performed using $\mathrm{R}$, version 2.15.2 ( $\mathrm{R}$ Core Team, R: A Language and Environment for Statistical Computing, Vienna, Austria: R Foundation for Statistical Computing; 2012, available at: http://www.R-project.org; "nonrandom": Susanne Stampf, nonrandom: Stratification and Matching by the Propensity Score, R Package, version 1.4, 2012. Q3, available at: http://CRAN.R-project.org/ package=nonrandom; "survival": Terry Therneau 2012, A Package for Survival Analysis in S, R package, version 2.36-14; and "rms": Frank E. Harrell, Jr, 2013; rms: Regression Modeling Strategies, R package, version 4.0-0, available at: http://CRAN.R-project.org/package=rms) packages were used.

\section{RESULTS}

A total of 1033 patients (303 MIDCAB and 730 DES-PCI) for isolated proximal LAD disease were 
TABLE 1. Baseline characteristics in unmatched and matched sample

\begin{tabular}{|c|c|c|c|c|c|}
\hline Characteristic & $\begin{array}{l}\text { MIDCAB unmatched } \\
(\mathbf{n}=\mathbf{3 0 3})\end{array}$ & $\begin{array}{l}\text { DES-PCI unmatched } \\
(\mathbf{n}=\mathbf{7 3 0})\end{array}$ & $\begin{array}{l}\text { SD before } \\
\text { matching }\end{array}$ & $\begin{array}{l}\text { DES-PCI matched } \\
\quad(\mathbf{n}=\mathbf{3 0 3})\end{array}$ & $\begin{array}{l}\text { SD after } \\
\text { matching }\end{array}$ \\
\hline Age $\geq 70 y$ & 85 (28.05) & $251(34.38)$ & 13.5 & $93(30.69)$ & 5.7 \\
\hline Female gender & $52(17.16)$ & $192(26.30)$ & 22 & $53(17.49)$ & 1.7 \\
\hline Hypertension & $209(68.98)$ & $391(53.56)$ & 39.5 & $191(63.04)$ & 6.1 \\
\hline Hypercholesterolemia & $247(81.52)$ & $464(63.56)$ & 43 & $227(74.92)$ & 7.2 \\
\hline NYHA class III-IV & $77(25.41)$ & $166(22.74)$ & 0.7 & $76(25.08)$ & 1.1 \\
\hline Previous MI & $89(29.37)$ & $172(23.56)$ & 13.6 & $80(26.40)$ & 6.6 \\
\hline Previous PCI & 79 (26.07) & $78(10.68)$ & 40.6 & $76(25.08)$ & 2.2 \\
\hline Current smokers & $23(7.59)$ & $106(14.52)$ & 13.7 & $37(12.21)$ & 9.5 \\
\hline Previous CVA & $15(4.95)$ & $6(0.82)$ & 30 & $5(1.65)$ & 9.1 \\
\hline PVD & $12(3.96)$ & $2(0.27)$ & 24 & $5(1.65)$ & 8.9 \\
\hline $\mathrm{LVEF}<50 \%$ & $16(5.28)$ & $96(13.15)$ & 19.2 & $24(7.92)$ & 4.6 \\
\hline $\mathrm{LVEF}<30 \%$ & $2(0.66)$ & $21(2.88)$ & 5.1 & $3(0.99)$ & 5.2 \\
\hline Diabetes & $58(19.14)$ & $137(18.77)$ & 7.5 & $45(14.85)$ & 2.7 \\
\hline Diabetes requiring insulin & $14(4.62)$ & $39(5.34)$ & 7.3 & $14(4.62)$ & 0.9 \\
\hline Creatinine $\geq 200 \mathrm{mmol} / \mathrm{L}$ & $3(0.99)$ & $20(2.74)$ & 7.1 & $10(3.30)$ & 9.2 \\
\hline Elective indication & $261(86.14)$ & $552(75.62)$ & 20.9 & $265(87.46)$ & 3.9 \\
\hline $\mathrm{BMI}>30 \mathrm{~kg} / \mathrm{m}^{2}$ & 79 (26.07) & $216(29.59)$ & 2.1 & 85 (28.05) & 1.3 \\
\hline Chronic total occlusion & 89 (23.37) & $60(8.21)$ & 39.4 & $55(18.15)$ & 8.1 \\
\hline
\end{tabular}

Data presented as n (\%). SD, Standardized difference in mean; NYHA, New York Heart Association; $M I$, myocardial infarction; $P C I$, percutaneous coronary intervention; $C V A$, cerebrovascular accident; $P V D$, peripheral vascular disease; $L V E F$, left ventricular ejection fraction; $B M I$, body mass index; $D E S-P C I$, percutaneous coronary intervention with drug-eluting stent; $M I D C A B$, minimally invasive direct coronary artery bypass.

included. The MIDCAB patients were more likely to have hypertension, hypercholesterolemia, a history of cerebrovascular accident, peripheral vascular disease, previous myocardial infarction, previous PCI, left ventricular ejection fraction $\leq 50 \%$, and more frequently had an elective indication. The prevalence of chronic total occlusion was significantly greater in the MIDCAB group.

The proportion of patients with age $\geq 70$ years was greater in the PCI group. The PCI patients were more frequently women, with a greater prevalence of current smokers.

No differences were found among the 2 groups in terms of the prevalence of functional New York Heart Association, diabetes mellitus, insulin therapy, prevalence of poor left ventricular function (left ventricular ejection fraction $<30 \%$ ), obesity, and baseline creatinine $\geq 200$ $\mathrm{mmol} / \mathrm{L}$.

Among the PCI patients, $499 \quad(68.3 \%)$ received sirolimus-eluting stents and $231(21.7 \%)$ paclitaxeleluting stents. The mean number of stents used was $1.2 \pm 0.48$.

All the initially observed differences were balanced in the 2 groups after matching, with standardized differences $<10$. Matching on the estimated PS made available a matched cohort of 606 patients, 303 for each group, with similar baseline risk factors. The patient characteristics before and after matching are listed in Table 1.

\section{Early Outcomes}

Overall, 5 of 1033 patients $(0.4 \%)$ died within 30 days from the index procedure, with no differences between the 2 groups (MIDCAB 2 of 303 [0.6\%] vs DES-PCI of 3 of $730[0.4 \%] ; P=.6$ ). A postoperative cerebrovascular accident occurred in 0 of 303 patients $(0 \%)$ and 1 of 730 patients $(0.13 \%)$ after MIDCAB and DES-PCI, respectively $(P=.9)$. Renal replacement therapy was required in 4 of 303 patients $(1.3 \%)$ and 10 of 730 patients $(1.3 \%)$ after MIDCAB and DES-PCI, respectively $(P=.9)$.

The incidence of local complications was 6 of 303 $(1.9 \%)$ and 15 of $730(2.0 \%)$ in the MIDCAB and DES-PCI groups, respectively $(P=.6)$. The median length of hospital stay was 5 days (range 5-7) and 1 day (range 1-1) in the MIDCAB and DES-PCI groups, respectively $(P<.001)$.

After matching, MIDCAB and DES-PCI presented with comparable 30-day mortality ( 2 of $303[0.6 \%]$ vs 1 of $303[0.3 \%] ; P=1.0$ ), postoperative cerebrovascular accident ( 0 of 303 vs 0 of 303; $P=.9)$, renal replacement therapy (4 of $303[1.2 \%]$ vs 4 of $303[1.2 \%] ; P=.7$ ), and local complications ( 6 of $303[1.8 \%]$ vs 6 of 303 $[1.8 \%] ; P=.8)$. The median length of hospital stay was longer in the MIDCAB group than in DES-PCI group (5 days; range, 5-7; and 1 day, range, $1-1 ; P<.001$ ).

\section{Follow-up and Late Outcomes}

The follow-up period ranged from 1 to 4449 days. The mean follow-up was $1979 \pm 1082$ days for all cases and $2232 \pm 1007$ days for the matched sample $(2286 \pm 1188$ days in the MIDCAB group and $2207 \pm 706$ days in the DES-PCI group).

Before matching, MIDCAB was associated with a significantly decreased risk of late death (unadjusted hazard 
TABLE 2. Effect of DES-PCI on late outcomes using MIDCAB as reference

\begin{tabular}{lllc}
\hline \multicolumn{1}{c}{ Outcomes } & $\boldsymbol{P}$ value & HR & 95\% CI of HR \\
\hline Death & .01 & 2.19 & $1.15-4.17$ \\
Repeat revascularization & .008 & 2.05 & $1.20-3.47$ \\
Death/RR & .0003 & 2.14 & $1.41-3.24$ \\
Sensitivity analysis according to age & & & \\
Death with age $\geq 70$ y & .02 & 2.73 & $1.17-6.38$ \\
Death with age $<70$ y & .5 & 1.40 & $0.50-3.93$ \\
RR with age $\geq 70$ y & .1 & 2.06 & $0.79-5.34$ \\
RR with age $<70$ y & .03 & 2.00 & $1.06-3.78$ \\
Death/RR with age $\geq 70$ y & .009 & 2.33 & $1.23-4.40$ \\
Death/RR with age $<70$ y & .01 & 1.93 & $1.11-3.34$ \\
Sensitivity analysis according to diabetes status & & \\
Death without diabetes & .019 & 2.5 & $1.16-5.50$ \\
Death with diabetes & .9 & 1.07 & $0.24-4.77$ \\
RR without diabetes & .016 & 2.14 & $1.15-3.98$ \\
RR with diabetes & .2 & 1.98 & $0.63-6.20$ \\
Death/RR with diabetes & .4 & 1.44 & $0.57-3.63$ \\
Death/RR without diabetes & .0006 & 2.36 & $1.44-3.87$ \\
Sensitivity analysis according to DES subgroup & & \\
Death with paclitaxel-ES & .06 & 2.12 & $0.95-4.74$ \\
Death with sirolimus-ES & .06 & 2.05 & $0.97-4.36$ \\
RR with paclitaxel-ES & .30 & 1.47 & $0.70-3.07$ \\
RR with sirolimus-ES & .04 & 1.89 & $1.02-3.51$ \\
Death/RR with paclitaxel-ES & .05 & 1.72 & $0.99-2.98$ \\
Death/RR with sirolimus-ES & .005 & 1.98 & $1.22-3.21$ \\
\hline DES $P$ CI Perctan
\end{tabular}

DES-PCI, Percutaneous coronary intervention with drug-eluting stent; $M I D C A B$, minimally invasive direct coronary artery bypass; $H R$, hazard ratio; $C I$, confidence interval; $R R$, repeat revascularization; $D E S$, drug-eluting stent; $E S$, eluting stent.

ratio [HR], 0.36; 95\% confidence interval [CI], 0.20-0.65; $P=.0008$ ), target vessel revascularization (unadjusted HR, 0.39; 95\% CI, 0.29-0.69; $P=.001)$, repeat revascularization (unadjusted HR, 0.44; 95\% CI, 0.27-0.71; $P=.001)$, and the composite of death or repeat revascularization (unadjusted $\mathrm{HR}, \quad 0.41 ; 95 \% \mathrm{CI}, \quad 0.28-0.60$; $P<.0001)$.

In the matched sample, 13 and 31 late deaths occurred in the MIDCAB and DES-PCI group, respectively $(P=.01)$. The survival rate in the MIDCAB and DES-PCI groups was $98.7 \pm 0.6$ versus $98.0 \pm 0.8$ at $1,95.4 \pm 1.3$ versus $93.3 \pm 1.5$ at 5 , and $94.8 \pm 1.3$ versus $93.3 \pm 1.3$ at 10 years of follow-up, respectively. Of the 303 patients in each group, $10(3.3 \%)$ versus $31(10.2 \%)$ required target vessel revascularization $(P=.009)$ and $20(6.6 \%)$ versus 43 $(14.2 \%)$ required repeat revascularization in MIDCAB and DES-PCI groups, respectively $(P=.006)$. The freedom from repeat revascularization in the 2 groups was $96.6 \pm 1.0$ versus $96.0 \pm 1.1$ at $1,93.2 \pm 1.5$ versus $86.8 \pm 2.0$ at 5 , and $91.8 \pm 1.8$ versus $86.8 \pm 2.0$ at 10 years of follow-up. DES-PCI was associated with a 2.19-fold increased risk of late death (95\% CI, 1.15-4.17), a 3.1-fold increased risk of target vessel revascularization $(95 \%$ CI, 1.35 4.21 ), a 2.0-fold increased risk of repeat revascularization (95\% CI, 1.20-3.47), and a 2.14-fold increased risk of the composite of death and repeat revascularization $(95 \% \mathrm{CI}$, 1.41-3.24).

MIDCAB was associated with better late outcomes regardless of age $\geq 70$ years, diabetes status, or type of drug-eluting stent used (Table 2, Figure 1).

The survival benefit from MIDCAB did not show a significant interaction with age $\left(\chi^{2}=0.19, P=.90\right)$, baseline serum creatinine $>200 \mathrm{mmol} / \mathrm{L}\left(\chi^{2}=3.5\right.$, $P=.06)$, and diabetes mellitus $\left(\chi^{2}=1.55, P=.21\right)$. A graphic representation of the hazard for late death suggested a trend toward reduced survival benefit from MIDCAB only in elderly patients with a baseline creatinine $>200 \mathrm{mmol} / \mathrm{L}$ (Figure 2).

\section{DISCUSSION}

The main finding of the present, single-center, 10-year, follow-up propensity score-matched comparison was that in patients with isolated proximal LAD disease, MIDCAB grafting LIMA to LAD was associated with a better longterm survival than DES-PCI. MIDCAB provided a survival advantage across all age classes, regardless of diabetes status or type of drug-eluting stent used. Only elderly patients with renal impairment were less likely to benefit from MIDCAB compared with DES-PCI in terms of late survival.

Although DES-PCI has been expected to reduce the need for repeat revascularization, DES-PCI to the proximal LAD has been still associated with a significant increased risk of repeat revascularization compared with MIDCAB, with a nonsignificant trend toward a better outcome when paclitaxel-eluting stents were used $(42 \%$ absolute risk reduction in the risk of repeat revascularization compared with sirolimus-eluting stents).

MIDCAB did not increase operative mortality and morbidity compared with DES-PCI. However, the length of hospital stay was increased among MIDCAB patients, as expected.

The optimal treatment of patients with isolated LAD disease continues to be debated.

MIDCAB has emerged as an attractive alternative to PCI, in particular for patients with isolated LAD stenosis, because it minimizes surgical trauma and is expected to provide better patency rate owing to the superiority of LIMA to LAD graft versus stenting. ${ }^{7}$

However, with the overall improvements in coronary stents and the introduction of DESs, interventional cardiologists and their patients have more often been choosing PCI as the initial treatment strategy for coronary revascularization, in particular for patients with isolated LAD stenosis. ${ }^{10}$ MIDCAB has emerged as an attractive alternative to conventional surgery in patients with isolated LAD stenosis to reduce surgical morbidity and provide excellent patency rate using LIMA to LAD graft. ${ }^{11}$ 

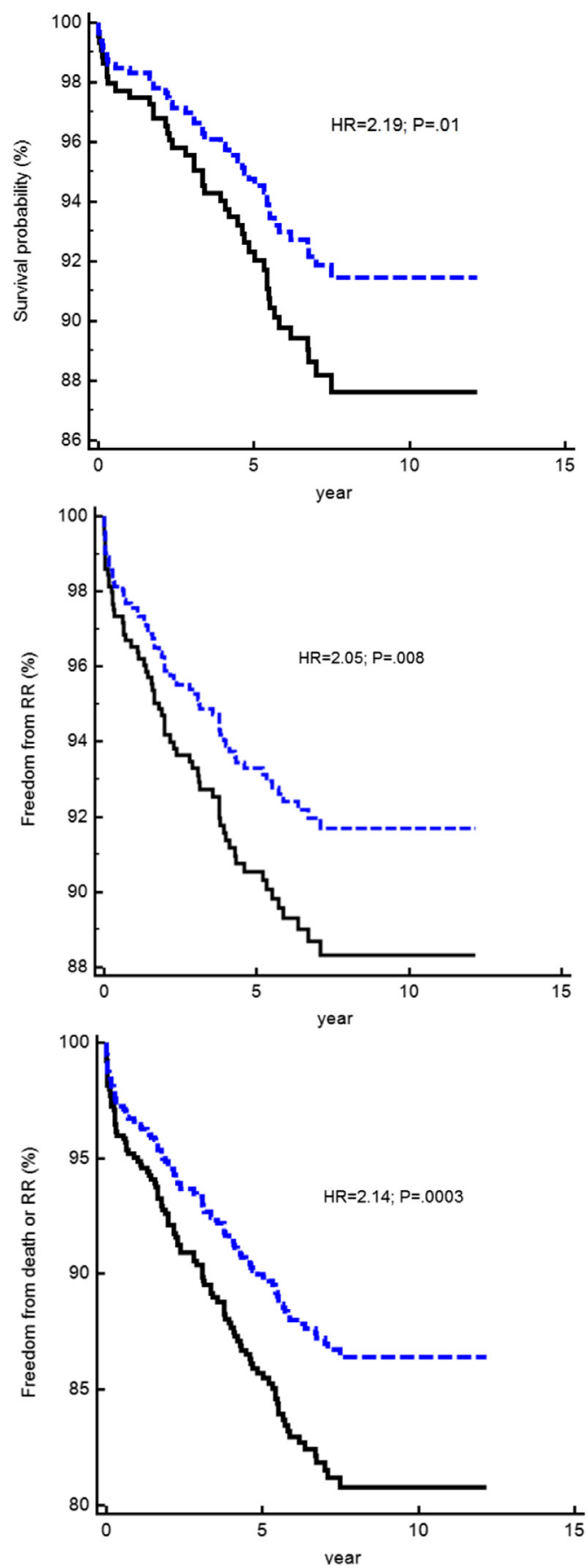
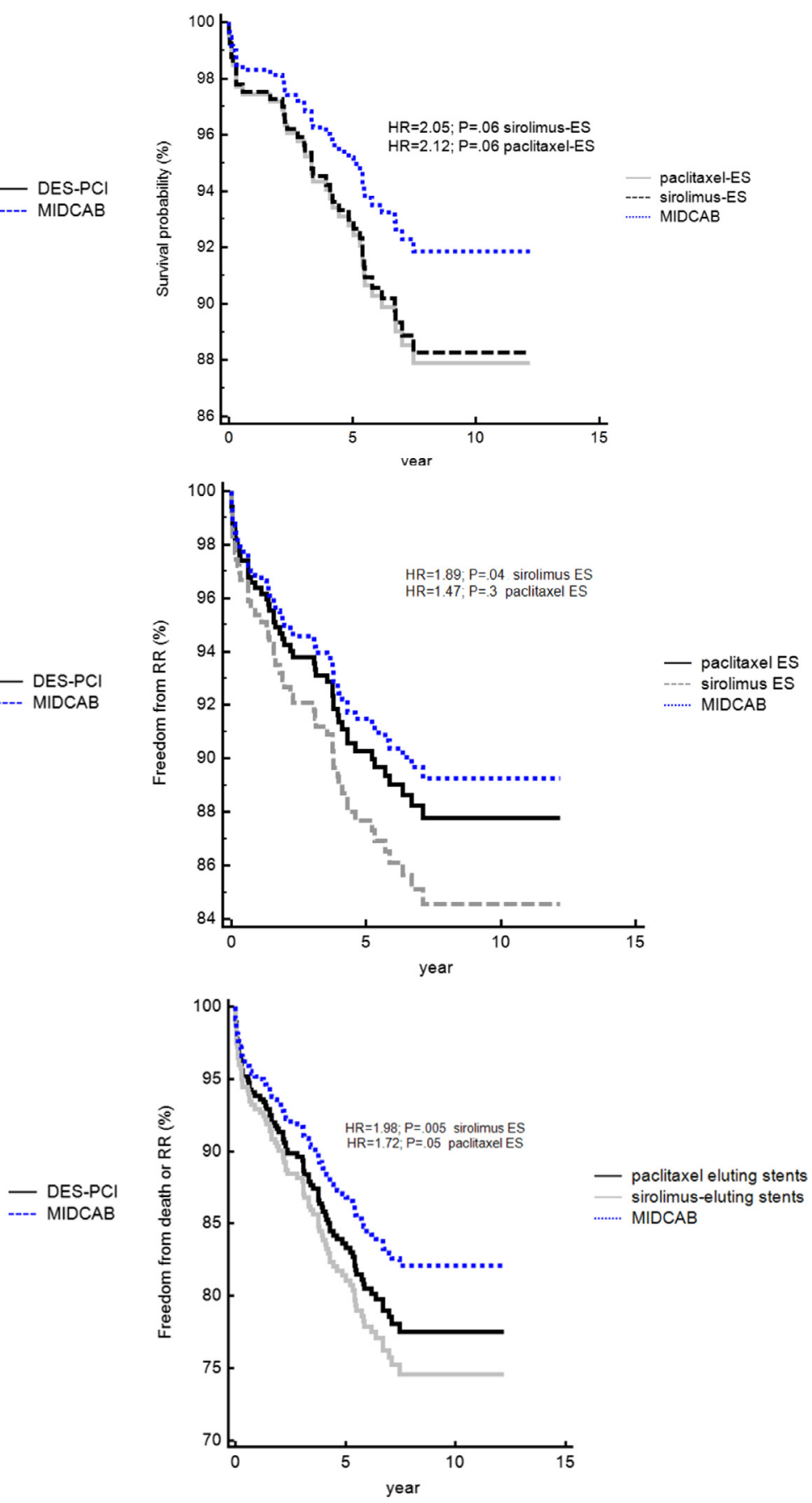

FIGURE 1. Survival curves according to Left, treatment and Right, type of drug-eluting stent implanted. HR, Hazard ratio; DES-PCI, percutaneous coronary intervention with drug-eluting stent; $M I D C A B$, minimally invasive direct coronary artery bypass; $E S$, eluting stent; $R R$, repeat revascularization.

Data comparing MIDCAB versus PCI are limited, and the lack of long-term follow-up remains a major limitation. A previous meta-analysis of a small number of randomized controlled trials by Jaffery and colleagues ${ }^{8}$ and Takagi and colleagues ${ }^{12}$ comparing MIDCAB and stenting showed PCI associated with an increased risk of repeat revascularization; however, the overall mortality was similar in the 2 groups. However, the study follow-up period was $\leq 1$ year for most of the studies. Only 1 small sample size trial by Hong and colleagues ${ }^{13}$ compared DES-PCI $(\mathrm{n}=119)$ and MIDCAB $(\mathrm{n}=70)$ and reported similar rates of a need for repeated revascularization and death (DES-PCI, $0.0 \%$ $[\mathrm{n}=0]$; vs MIDCAB, $2.9 \%[\mathrm{n}=2]$ ) during 6 months of follow-up. 


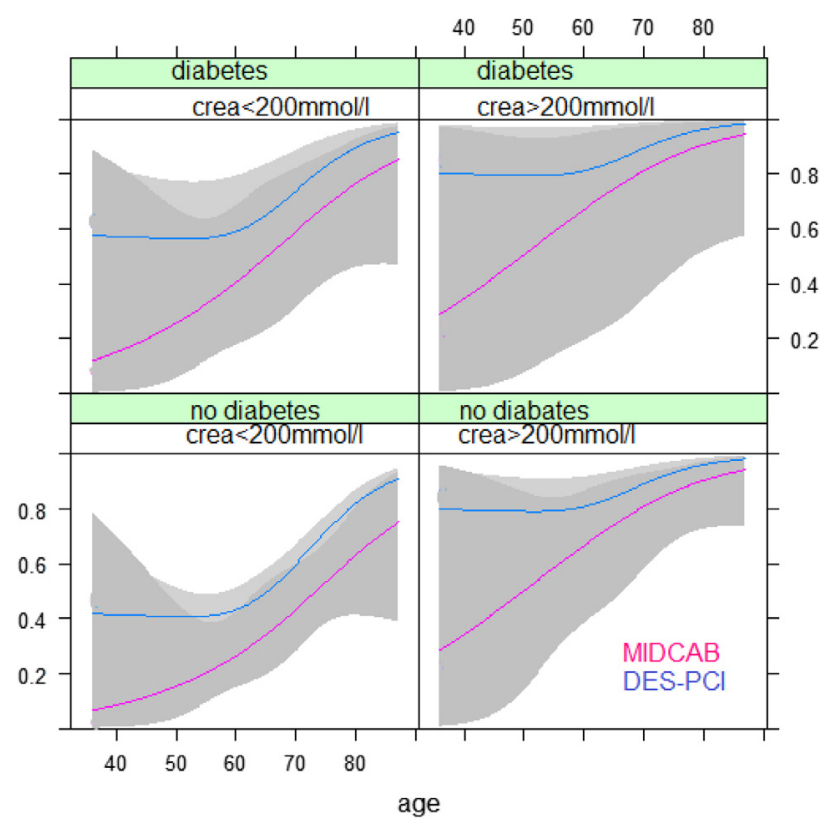

FIGURE 2. Probability for late death for minimally invasive direct coronary artery bypass $(M I D C A B)$ versus percutaneous coronary intervention with drug-eluting stents (DES-PCI) stratified by age, diabetes status, and creatinine $($ crea $)>200 \mathrm{mmol} / \mathrm{L}$.

It should be underlined that because target vessel revascularization was the most frequently occurring event and reflected the major difference between surgical and PCI therapy, these randomized trials were powered to detect differences in major adverse cardiac events inevitably failed to detect any differences in the more clinically significant endpoints of mortality and myocardial infarction owing to a lack of power.

Observational studies comparing DES-PCI and MID$\mathrm{CAB}$ have been scarce, with major limitations. Ben-Gal and colleagues ${ }^{14}$ compared 2 years of outcomes among 88 matched pairs of patients receiving DES-PCI (sirolimus) versus MIDCAB. DES-PCI was found to increase the risk of reintervention (HR, 8.26) but not 2-year death (2-year survival, $98 \%$ vs $93 \%$ in the DES-PCI vs MIDCAB group; $P=.11)$. However, the main indication for MIDCAB was to minimize surgical trauma in patients with 2-vessel or 3 -vessel disease. Thus, most of MIDCAB patients received incomplete revascularization, and this limitation could have significantly affected the MIDCAB outcomes. Another limitation of the present study was the relatively limited mean follow-up period of 22 months. Etienne and colleagues ${ }^{15}$ recently compared the 5 -year outcomes among 196 DES-PCI and 260 MIDACB unmatched, multicenter patients. They found that the freedom from the need for repeat revascularization was significantly in favor of the MIDCAB group (HR, 0.17; $P<.0001$ ) but the 5-year survival was similar after MIDCAB and DES-PCI $(P=.89)$. However, despite the significant difference between the 2 groups, the investigators were unable to perform PS matching because of the small sample size in the DES-PCI group. In addition, only a small number of patients undergoing MIDCAB (24\%) had had proximal LAD disease. It has been well demonstrated that the survival benefit from $\mathrm{CABG}$ is relevant in the presence of proximal LAD disease, and this aspect might have mitigated the expected survival benefit from MIDCAB compared with DES-PCI.

To the best of our knowledge, the present study is the largest sample size, single-center comparison of the longterm outcomes after MIDCAB versus DES-PCI. At 10 years of follow-up, DES-PCI had significantly increased the risk of late death compared with MIDCAB. DES-PCI was also associated with a significantly increased risk of repeat revascularization. The benefit from MIDCAB was present across all age classes and in the presence of diabetes.

Moreover, MIDCAB was not associated with increased operative morbidity and mortality compared with DESPCI for all outcomes investigated, including stroke. The risk of stroke has been consistently shown to be increased by CABG compared with PCI in patients with multivessel disease, and this has been related to the aortic manipulation required for proximal graft anastomosis or aortic cannulation. ${ }^{16}$ In contrast to conventional CABG, MIDCAB using an off-pump technique avoids any aortic manipulation.

In the present surgical cohort, most MIDCABs were performed by a single surgeon fully experienced in off-pump CABG, and this aspect might have contributed to the excellent outcomes with MIDCAB. However, the survival advantage from MIDCAB compared with DES-PCI could not be explained only by the reduction in the need for repeat revascularization. LIMA grafting has been shown to have a strong protective effect against progression of native coronary artery disease in previously grafted vessels. ${ }^{17}$ Therefore, MIDCAB can improve long-term survival by preventing progression of atherosclerosis in the native coronary vessels. In addition, evidence has been increasing that drug-eluting stents can develop delayed thrombosis related to delayed endothelialization, hypersensitivity to the stent polymer, or the discontinuation of antiplatelet treatment. ${ }^{18-20}$ This aspect might account for the increased risk of death among DES-PCI patients, regardless of the need for repeat revascularization.

The present study had limitations. This was a retrospective, single-center study analyzing the clinical outcomes during a 10-year period; thus, its conclusions might not be generalizable to other experiences, populations, or current practices. Also, despite the use of PS matching as one of the most robust tools (other than a randomized controlled trial) to compare treatment effects, this was a retrospective analysis with the known inherent limitations of patient selection and treatment bias. Hidden biases have often been claimed as an explanation for the apparent superiority 
of MIDCAB compared with DES-PCI. According to this claim, clinicians have allocated patients with a poor prognosis to PCI, but the measured covariates have not captured this bias. Factors precluding MIDCAB have included coexisting conditions linked to a poor prognosis, such as malignancy, and the factors contraindicating PCI have often been "lesion based," such as chronic vessel occlusion, and could have a smaller effect on the longterm outcomes after CABG.

\section{CONCLUSIONS}

The expected diminution of restenosis with DES and the lack of evidence for a survival benefit from MIDCAB have led to even more widespread adoption of percutaneous techniques instead of MIDCAB in the treatment of isolated proximal LAD disease. The present findings have demonstrated a long-term survival benefit and reduced need for reintervention from MIDCAB compared with DES-PCI in a "real world" clinical practice. According to our results, MIDCAB should not only be offered in the presence of isolated proximal LAD disease unsuitable for PCI, but should also be considered as a first-line treatment in this setting. These results advocate for additional wellpowered randomized controlled trials with long-term follow-up data to define the optimal treatment of isolated proximal LAD disease.

\section{References}

1. Hakeem A, Garg N, Bhatti S, Rajpurohit N, Ahmed Z, Uretsky BF. Effectiveness of percutaneous coronary intervention with drug-eluting stents compared with bypass surgery in diabetics with multivessel coronary disease: comprehensive systematic review and meta-analysis of randomized clinical data. J Am Heart Assoc. 2013;2:e00354.

2. Mohr FW, Morice MC, Kappetein AP, Feldman TE, Ståhle E, Colombo A, et al. Coronary artery bypass graft surgery versus percutaneous coronary intervention in patients with three-vessel disease and left main coronary disease: 5-year follow-up of the randomised, clinical SYNTAX trial. Lancet. 2013;381:629-38.

3. Benedetto U, Melina G, Angeloni E, Refice S, Roscitano A, Fiorani B, et al. Coronary artery bypass grafting versus drug-eluting stents in multivessel coronary disease: a meta-analysis on 24,268 patients. Eur $J$ Cardiothorac Surg. 2009;36:611-5.

4. Daemen J, Boersma E, Flather M, Booth J, Stables R, Rodriguez A, et al. Long-term safety and efficacy of percutaneous coronary intervention with stenting and coronary artery bypass surgery for multivessel coronary artery disease: a meta-analysis with 5-year patient-level data from the ARTS, ERACI-II, MASS-II, and SoS trials. Circulation. 2008;118:1146-54.
5. Thiele H, Falk V. Coronary artery bypass graft versus drug-eluting stent for highrisk proximal left anterior descending stenosis. Curr Treat Options Cardiovasc Med. 2010;12:36-45.

6. Thiele H, Neumann-Schniedewind P, Jacobs S, Boudriot E, Walther T, Mohr FW, et al. Randomized comparison of minimally invasive direct coronary artery bypass surgery versus sirolimus-eluting stenting in isolated proximal left anterior descending coronary artery stenosis. J Am Coll Cardiol. 2009;53:2324-31.

7. Calafiore AM, Teodori G, Di Giammarco G, Vitolla G, Iaco’ A, Iovino T, et al. Minimally invasive coronary artery bypass grafting on a beating heart. Ann Thorac Surg. 1997;63(6 suppl):S72-5.

8. Jaffery Z, Kowalski M, Weaver WD, Khanal S. A meta-analysis of randomized control trials comparing minimally invasive direct coronary bypass grafting versus percutaneous coronary intervention for stenosis of the proximal left anterior descending artery. Eur J Cardiothorac Surg. 2007;31:691-7.

9. Austin PC. Propensity-score matching in the cardiovascular surgery literature from 2004 to 2006: a systematic review and suggestions for improvement. J Thorac Cardiovasc Surg. 2007;134:1128-35.

10. Sawhney N, Moses JW, Leon MB, Kuntz RE, Popma JJ, Bachinsky W, et al. Treatment of left anterior descending coronary artery disease with sirolimuseluting stents. Circulation. 2004;110:374-9.

11. Diegeler A, Spyrantis N, Matin M, Falk V, Hambrecht R, Autschbach R, et al. The revival of surgical treatment for isolated proximal high grade LAD lesions by minimally invasive coronary artery bypass grafting. Eur J Cardiothorac Surg. 2000;17:501-4.

12. Takagi H, Tanabashi T, Kawai N, Umemoto T. A meta-analysis of minimally invasive coronary artery bypass versus percutaneous coronary intervention with stenting for isolated left anterior descending artery disease is indispensable. J Thorac Cardiovasc Surg. 2007;134:548-9.

13. Hong SJ, Lim DS, Seo HS, Kim YH, Shim WJ, Park CG, et al. Percutaneous coronary intervention with drug-eluting stent implantation vs. minimally invasive direct coronary artery bypass (MIDCAB) in patients with left anterior descending coronary artery stenosis. Catheter Cardiovasc Interv. 2005;64:75-81.

14. Ben-Gal Y, Mohr R, Braunstein R, Finkelstein A, Hansson N, Hendler A, et al. Revascularization of left anterior descending artery with drug-eluting stents: comparison with minimally invasive direct coronary artery bypass surgery. Ann Thorac Surg. 2006;82:2067-71.

15. Etienne PY, D'hoore W, Papadatos S, Mairy Y, El Khoury G, Noirhomme P, et al. Five-year follow-up of drug-eluting stents implantation vs minimally invasive direct coronary artery bypass for left anterior descending artery disease: a propensity score analysis. Eur J Cardiothorac Surg. 2013;44:884-90.

16. Marui A, Kimura T, Tanaka S, Okabayashi H, Komiya T, Furukawa Y, et al; CREDO-Kyoto Investigators. Comparison of frequency of operative stroke in off-pump coronary artery bypass grafting versus on pump coronary artery bypass grafting versus percutaneous coronary intervention. Am J Cardiol. 2012;110: $1773-8$.

17. Dimitrova KR, Hoffman DM, Geller CM, Dincheva G, Ko W, Tranbaugh RF. Arterial grafts protect the native coronary vessels from atherosclerotic disease progression. Ann Thorac Surg. 2012;94:475-81.

18. Kimura T, Morimoto T, Nakagawa Y, Kawai K, Miyazaki S, Muramatsu T, et al. Very late stent thrombosis and late target lesion revascularization after sirolimus-eluting stent implantation: five-year outcome of the J-Cypher registry. Circulation. 2012;125:584-91

19. Wenaweser P, Daemen J, Zwahlen M, van Domburg R, Jüni P, Vaina S, et al. Incidence and correlates of drug-eluting stent thrombosis in routine clinical practice: 4-year results from a large 2-institutional cohort study. J Am Coll Cardiol. 2008;52:1134-40.

20. Raja SG, Berg GA. Safety of drug eluting stents: current concerns and controversies. Curr Drug Saf. 2007;2:212-9. 